\title{
Paradigm shifts in solar dynamo modeling
}

\author{
Axel Brandenburg \\ Nordita, Roslagstullsbacken 23, 10691 Stockholm, Sweden
}

\begin{abstract}
Selected topics in solar dynamo theory are being highlighted. The possible relevance of the near-surface shear layer is discussed. The role of turbulent downward pumping is mentioned in connection with earlier concerns that a dynamo-generated magnetic field would be rapidly lost from the convection zone by magnetic buoyancy. It is argued that shear-mediated small-scale magnetic helicity fluxes are responsible for the success of some of the recent largescale dynamo simulations. These fluxes help in disposing of excess small-scale magnetic helicity. This small-scale magnetic helicity, in turn, is generated in response to the production of an overall tilt in each Parker loop. Some preliminary calculations of this helicity flux are presented for a system with uniform shear. In the Sun the effects of magnetic helicity fluxes may be seen in coronal mass ejections shedding large amounts of magnetic helicity.
\end{abstract}

Keywords. MHD - turbulence - Sun: coronal mass ejections (CMEs) - Sun: magnetic fields

\section{Introduction}

Unlike the geodynamo, which is widely regarded a solved problem (e.g. Glatzmaier \& Roberts 1995), the solar dynamo problem remains unsolved in that there is no single model that is free of theoretical shortcomings and that actually reproduces the Sun. The flux-transport dynamo models (e.g. Dikpati \& Charbonneau 1999; Küker et al. 2001; Chatterjee et al. 2004) are currently the only models that display some degree of realism, but there are aspects in their design that are arguably not sufficiently plausible. One problem is the assumption of a rather low turbulent magnetic diffusivity that needs to be assumed in a ad hoc fashion. On theoretical grounds the turbulent magnetic diffusivity should be comparable with the turbulent kinematic viscosity (Yousef et al. 2003), which is not the case on these flux-transport dynamo models.

The purpose of this review is to collect recent findings that are relevant in solving the solar dynamo problem. On the one hand, there are direct simulations of convective dynamo action in spherical shells by Brun \& Toomre (2002), Brun et al. (2004, 2006), Browning et al. (2006), and Brown et al. (2007). These models are already quite realistic and should ultimately be able to reproduce the solar dynamo. Their main shortcomings range from still insufficient resolution (even though it is already exhausting current capabilities) to the negligence of certain physical features of the model. Examples of shortcomings include the still insufficient degree of stratification as well as the simplified treatment or even the neglect of surface and tachocline boundary layers. On the other extreme, there are phenomenologically motivated mean-field models that are constructed based on their success in reproducing the Sun. These models tend to utilize a subset of known turbulent transport effects with ad hoc amplitudes and prescriptions of their radial profiles. Such models are made nonlinear by simple $\alpha$ quenching terms. However, this type of prescription that is still commonly used in mean field models does not correctly describe the saturation behavior of large-scale magnetic fields as known from three-dimensional turbulence simulations. This is true even for much simpler models, for example those where the turbulence is driven at a specific length scale via a forcing function (Brandenburg 2001). 

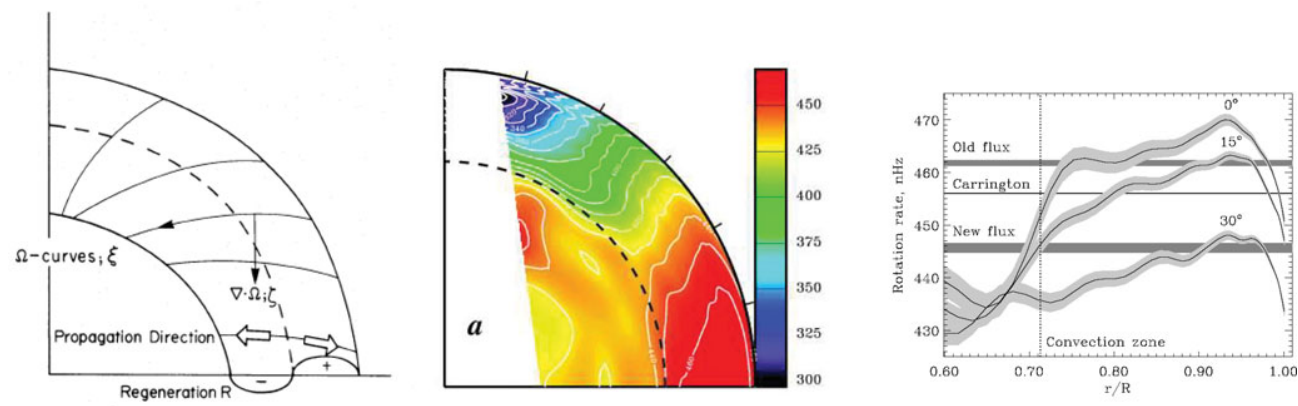

Figure 1. Comparison of the differential rotation contours that were originally expected by Yoshimura (1975) based on solar dynamo model considerations (left) with those by Thompson et al. (2003) using helioseismology (middle). Note the similarities between the contours on the left (over the bulk of the convection zone) and those on the right (over the outer $5 \%$ of the solar radius). In the right hand panel we show radial profiles of angular velocity as given by Benevolenskaya et al. (1999). Note the sharp negative radial gradient near the surface.

\section{Negative radial shear?}

In the 1970s a number of solar dynamo models were discussed that were based on an $\alpha$ effect that is positive in the northern hemisphere (and negative in the southern hemisphere) and a differential rotation that had a negative radial gradient, i.e. $\partial \Omega / \partial r<0$ (see the left panel of Fig. 1). Such models produce an equatorward migration of magnetic activity toward the equator. Examples of such models in full spherical geometry include the papers Steenbeck \& Krause (1969), Roberts \& Stix (1972), Köhler (1973), and Yoshimura (1975). During the 1980s these models faced two difficulties, a conceptual one and one based on a conflict with observations. The conceptual difficulty has to do with the idea that the magnetic field may be fibral (Parker 1984), i.e. it is distributed in the form of many flux tubes with a small filling factor and relatively large field strength. However, such fields would easily be unstable to magnetic buoyancy instabilities. This and other considerations led Spiegel \& Weiss (1980) to propose that the solar dynamo might instead work at the bottom of the solar convection zone and not, as assumed until them, in a distributed fashion in the bulk of the convection zone. In fact, in an earlier paper by Parker (1975) this possibility was already discussed in some detail. The conflict with observations had to do with the fact that helioseismology began to put tight constraints on the form of the Sun's internal angular velocity profile. This seemed to rule out a negative radial $\Omega$ gradient.

These two difficulties led to a new type of dynamo model that works in the lower overshoot layer, where $\alpha$ has the opposite sign (DeLuca \& Gilman 1986, 1988). Not only would magnetic flux tubes presumably be stabilized against magnetic buoyancy instabilities, but at that depth the sign of the $\alpha$ effect would be reversed and thus dynamo waves would again propagate equatorward even though $\partial \Omega / \partial r>0$. However, several problems with this approach have been discussed (phase relation, number of toroidal flux belts, etc.). Yet another model is the flux transport model, where a meridional circulation (equatorward at the bottom of the convection zone) is chiefly responsible for driving the dynamo wave equatorward. However, it is now quite clear that there is a strong shear layer near the surface where $\partial \Omega / \partial r<0$, just as originally anticipated. The difference is that now this shear layer only extends over the outer $5 \%$ of the solar radius. In the past the relevance of this near-surface shear layer was discarded mainly on the grounds that magnetic buoyancy effects would lead to a rapid loss of magnetic field. Another reason is that near the surface the local turnover time is still short compared 

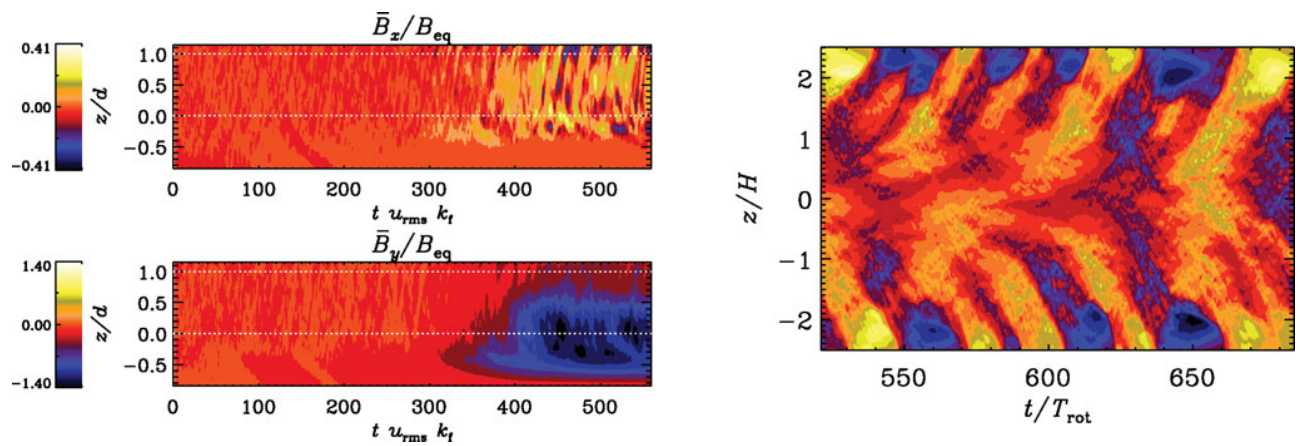

Figure 2. Horizontally averaged magnetic fields $\bar{B}_{x}$ (upper panel on the left) and $\bar{B}_{y}$ (lower panel on the left), as functions of time and $z$ for a run with shear and no rotation (Käpylä et al. 2008 ), as well as $\bar{B}_{y}$ from a local model of an accretion disc (right hand panel), where $z=0$ corresponds to the midplane. On the left-hand panel the dotted white lines show top $(z=d)$ and bottom $(z=0)$ of the convection zone.

with the rotation period. However, simulations of compressible convection did show some time ago that magnetic buoyancy is effectively overpowered by the effects of turbulent downward pumping. Regarding the timescales, rotational effects are likely to play a role at the bottom of the near-surface shear layer, which is at a depth of about $40 \mathrm{Mm}$. The relative importance of rotation and convection is determined by the Coriolis number,

$$
\mathrm{Co}=2 \Omega \tau,
$$

where $\Omega$ is the solar rotation rate and $\tau=H_{p} / u_{\mathrm{rms}}$ is the turnover time with $H_{p}$ being the pressure scale height. Using $\Omega=3 \times 10^{-6} \mathrm{~s}^{-1}$ for the solar rotation rate, $u_{\mathrm{rms}}=50 \mathrm{~m} / \mathrm{s}$ for the rms velocity at a depth of $40 \mathrm{Mm}$, and $H_{p}=13 \mathrm{Mm}$ for the pressure scale height at that depth we find $\tau=1.3 \mathrm{~d}$, and hence $\mathrm{Co}=1.4$, suggesting that rotation should become important at the bottom of the near-surface shear layer.

\section{Quenching and examples of large-scale dynamos}

Since the early 1990s there has been the notion that catastrophic $\alpha$ quenching has been a major concern in dynamo theory (Vainshtein \& Cattaneo 1992). This led Parker (1993) to suggest that the solar dynamo might work in a segregated fashion where shear acts only near the lower overshoot layer where the turbulent magnetic diffusivity is low. In the bulk of the convection zone, on the other hand, the magnetic field is supposed to be weak, and so there was hope that the $\alpha$ effect would not be catastrophically quenched. However, only later the origin of catastrophic quenching was understood to be due to magnetic helicity conservation. As a consequence, catastrophic quenching could not be alleviated by spatial rearrangements, but only by magnetic helicity fluxes out of the domain. Indeed, there are now a number of simulations that show successful generation of large-scale magnetic fields; see Fig. 2. All these simulations have in common that there are contours of constant shear crossing the outer surface. This is believed to be important because the magnetic helicity flux is expected to be directed along the contours of constant shear (Brandenburg \& Subramanian 2005a).

\section{4. $\alpha$ effect and production of small-scale magnetic helicity}

The $\alpha$ effect is central to many mean-field approaches of the solar dynamo. Its presence is usually associated with the systematic action of the Coriolis force on convecting 

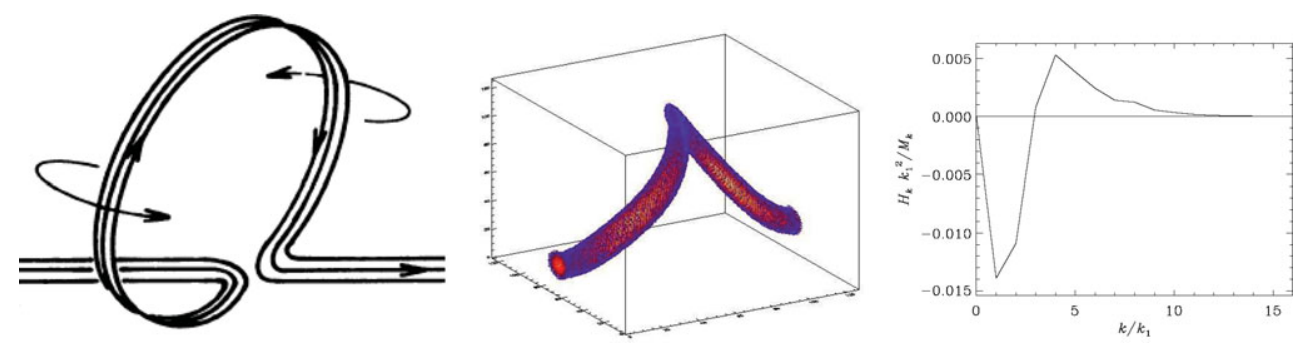

Figure 3. Original sketch of a Parker loop (Parker 1957) on the left, compared with a loop constructed from the Cauchy solution of Yousef \& Brandenburg (2003) and its corresponding magnetic helicity spectrum $H_{k}$ on the right.

fluid elements. As such an element rises vertically, it expands and counter rotates due to the Coriolis force, thereby attaining a downward oriented vorticity in the northern hemisphere, and an upward oriented vorticity in the southern hemisphere. As the magnetic field is dragged along with the gas, a systematic poloidal field component is being produced from a toroidal field. An early sketch of this process was given by Parker (1957), where a rising loop attains a systematic tilt; see the left hand panel of Fig. 3.

In recent years the nonlinear saturation of this process has been understood to be due to the internal twist that is being induced as the loop begins to tilt. This is already quite evident from the early sketch by Parker (1957) suggesting the presence of a spatially extended structure of the loop with oppositely oriented twisting motions on both ends of the loop; see the round arrows on the tube in the left hand panel of Fig. 3.

In the middle panel of Fig. 3 we show a visualization of an analytically generated representation of a Parker loop using the Cauchy solution for ideal magnetohydrodynamics together with the corresponding numerically constructed magnetic helicity spectrum (Yousef \& Brandenburg 2003); see the right hand panel of Fig. 3. This spectrum shows quite clearly a negative magnetic helicity at low wavenumbers $k / k_{1} \approx 1$ (where $k_{1}=2 \pi / L$ is the smallest wavenumber in a box of scale $L$ ) and positive magnetic helicity at larger wavenumbers around $k / k_{1} \approx 4$. This confirms an early finding of Seehafer (1996) that the $\alpha$ effect corresponds to a segregation of magnetic helicity in scale and that magnetic helicity of smaller scale and opposite sign is inevitably being produced by the $\alpha$ effect. It is this what quenches the $\alpha$ effect in a potentially catastrophic manner. Alleviating this type of quenching requires that we have to get rid of this smaller-scale magnetic helicity through helicity fluxes. This is something that the Sun seems to accomplish through coronal mass ejections (Démoulin et al. 2002, Blackman \& Brandenburg 2003). In the following we describe in more detail the magnetic helicity flux and its relation to the amount of shear.

\section{Magnetic helicity flux}

There are a number of analytic calculations of magnetic helicity fluxes relevant to astrophysical dynamos (Kleeorin et al. 2000, 2002, 2003a,b; Vishniac \& Cho 2001; Subramanian \& Brandenburg 2004, 2006; Brandenburg \& Subramanian 2005a,b). Here we present preliminary numerical calculations in a simple system. We calculate the smallscale current helicity flux, $\overline{\mathcal{F}}_{C}^{\mathrm{SS}}$, in homogeneous turbulence in the presence of shear, $S$, and a uniform magnetic field $\boldsymbol{B}_{0}$. Since the system is completely uniform, it makes sense to consider full volume averages, denoted here by an overbar. The wavenumber of the averaged quantities is zero, so we have infinite scale separation and can then force the turbulence at the scale of the system, i.e. at wavenumbers between 1 and 2 times the 

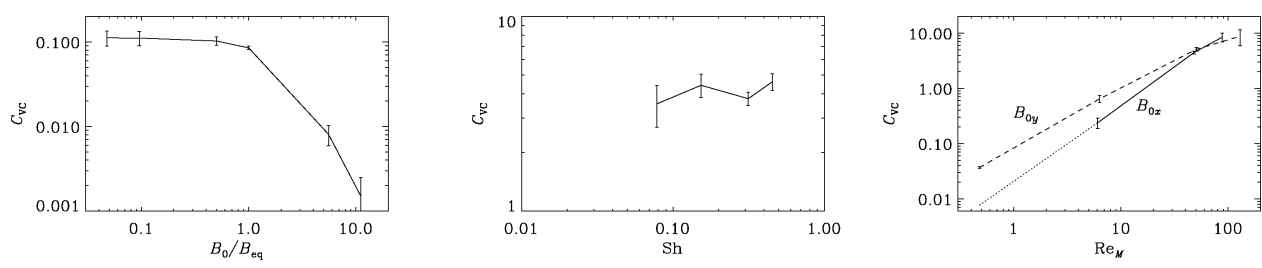

Figure 4. $B$ dependence of $-C_{\mathrm{VC}}$ for $\boldsymbol{B}_{0}=B_{0 y} \hat{\boldsymbol{y}}$ with $R_{\mathrm{m}}=1.4$, Sh $\approx-2$ (left), $S$ dependence of $C_{\mathrm{VC}}$ for $R_{\mathrm{m}}=30, B_{0} / B_{\mathrm{eq}} \approx 0.2$ (middle), and $R_{\mathrm{m}}$ dependence of $C_{\mathrm{VC}}$ for $\mathrm{Sh} \approx 0.5$, $B_{0} / B_{\mathrm{eq}} \approx 0.2$.

smallest finite wavenumber, $k_{1}$. Our average forcing wavenumber is therefore $k_{\mathrm{f}} / k_{1}=1.5$. We consider the dependence on the parameters $B_{0} / B_{\text {eq }}, R_{\mathrm{m}}$ and $\mathrm{Sh}$, where

$$
B_{\mathrm{eq}}^{2}=\mu_{0}\left\langle\rho \boldsymbol{u}^{2}\right\rangle, \quad R_{\mathrm{m}}=u_{\mathrm{rms}} / \eta k_{\mathrm{f}}, \quad \mathrm{Sh}=S / \eta k_{\mathrm{f}}^{2} .
$$

The small-scale current helicity flux is calculated from the expression (Brandenburg \& Subramanian 2005b)

$$
\overline{\mathcal{F}}_{C}^{\mathrm{SS}}=2 \overline{\boldsymbol{e} \times \boldsymbol{j}}+\overline{(\boldsymbol{\nabla} \times \boldsymbol{e}) \times \boldsymbol{b}},
$$

where

$$
\boldsymbol{e}=-\boldsymbol{u} \times\left(\boldsymbol{b}+\boldsymbol{B}_{0}\right)+\eta \mu_{0} \boldsymbol{j}
$$

is the small-scale electric field and $\boldsymbol{j}=\boldsymbol{\nabla} \times \boldsymbol{b} / \mu_{0}$ is the small-scale current density with $\mu_{0}$ being the vacuum permeability. In order to avoid taking more than two derivatives we integrate the second expression in equation (5.2) by parts, i.e.

$$
\overline{(\boldsymbol{\nabla} \times \boldsymbol{e}) \times \boldsymbol{b}}=\overline{(\boldsymbol{\nabla} \boldsymbol{b})^{T} \boldsymbol{e}}
$$

where we have used the fact that $\boldsymbol{\nabla} \boldsymbol{b}=0$. The $i$ component of this term can also be written as $\overline{b_{j, i} e_{j}}$, where a comma denotes partial differentiation.

Based on calculations using the minimal $\tau$ approximation the small-scale current helicity flux is expected to be given by (Brandenburg \& Subramanian 2005a)

$$
\overline{\mathcal{F}}_{C}^{\mathrm{SS}}=C_{\mathrm{VC}}(\overline{\mathbf{S B}}) \times \overline{\boldsymbol{B}},
$$

where $C_{\mathrm{VC}}$ is a non-dimensional coefficient that is of the order of $\mathrm{St}^{2}$, where $\mathrm{St}=\tau u_{\mathrm{rms}} k_{\mathrm{f}}$ is the Strouhal number. Throughout this work we use a uniform shear flow, i.e. $\overline{\boldsymbol{U}}=$ $(0, S x, 0)$, so the $z$ component of $\overline{\mathcal{F}}_{C}^{\text {SS }}$ is

$$
\overline{\mathcal{F}}_{C z}^{\mathrm{SS}}=\frac{1}{2} C_{\mathrm{VC}} S\left(\bar{B}_{y}^{2}-\bar{B}_{x}^{2}\right) .
$$

In the following we quote values of $C_{\mathrm{VC}}$ that are computed by imposing a uniform field either in the $x$ or in the $y$ direction. The numerical resolution is only $32^{3}$. In the left hand panel of Fig. 4 we show the dependence on $B_{0}$ for a small value of $R_{\mathrm{m}}\left(R_{\mathrm{m}}=1.4\right)$ and fixed shear parameter $\mathrm{Sh} \approx-2$ (for $S<0$ ). So far there is no indication that the flux depends on Sh; see the middle panel of Fig. 4. However, the flux shows a strong increase with $R_{\mathrm{m}}$; see the right hand panel of Fig. 4 . It should be noted that, since we keep the forcing unchanged while changing the viscosity, the resulting rms velocity also changes, and so the resulting values of Sh and $B_{0} / B_{\text {eq }}$ also change somewhat.

\section{Discussion}

In this paper we have presented selected aspects of the solar dynamo problem where there has been recent progress. The idea that the solar dynamo may operate in the bulk 
of the convection zone is motivated in part by the fact that it is easier to dispose of small-scale magnetic helicity from upper layers than from deeper down. Also the sign of the radial differential rotation is negative and would produce equatorward migration of dynamo waves in the presence of a positive $\alpha$ effect in the northern hemisphere of the Sun. A number of other arguments for a distributed solar dynamo, where the field that makes sunspots does not solely come from the lower overshoot layer, have been discussed elsewhere (Brandenburg 2005). However, what is not yet well addressed is the production of sunspots within active regions, and perhaps even the active regions themselves. It is plausible, and it has indeed been argued, that sunspots can be the result of negative turbulent magnetic pressure effects (Kleeorin \& Rogachevskii 1994) or a turbulent magnetic collapse phenomenon (Kitchatinov \& Mazur 2000). Both processes rely on turbulent transport processes that could be verified numerically and whose effects could also be demonstrated directly in suitably arranged simulations.

\section{Acknowledgements}

The computations have been carried out at the National Supercomputer Centre in Linköping and at the Center for Parallel Computers at the Royal Institute of Technology in Sweden. This work was supported in part by the Swedish Research Council.

\section{References}

Benevolenskaya, E. E., Hoeksema, J. T., Kosovichev, A. G., \& Scherrer, P. H. 1999, ApJ 517, L163

Blackman, E. G. \& Brandenburg, A. 2003, ApJ 584, L99

Brandenburg, A. 2001, ApJ 550, 824

Brandenburg, A. 2005, ApJ 625, 539

Brandenburg, A. \& Subramanian, K. 2005a, AN 326, 400

Brandenburg, A. \& Subramanian, K. 2005b, Phys. Rep., 417, 1

Brown, B. P., Browning, M. K., Brun, A. S., et al. 2007, AIPC, 948, 271

Browning, M. K., Miesch, M. S., Brun, A. S., \& Toomre, J. 2006, ApJ 648, L157

Brun, A. S. \& Toomre, J. 2002, ApJ 570, 865

Brun, A. S., Miesch, M. S. \& Toomre, J. 2004, ApJ 614, 1073

Brun, A., Miesch, M., \& Toomre, J. 2006, ApJ, 614, 1073

Chatterjee, P., Nandy, D., \& Choudhuri, A. R. 2004, A\& A 427, 1019

DeLuca, E. E., Gilman, P. A. 1986, Geophys. Astrophys. Fluid Dyn. 37, 85

DeLuca, E. E., Gilman, P. A. 1988, Geophys. Astrophys. Fluid Dyn. 43, 119

Démoulin, P., Mandrini, C. H., van Driel-Gesztelyi, L., et al. 2002, ApJ 382, 650

Dikpati, M. \& Charbonneau, P. 1999, ApJ 518, 508

Glatzmaier, G. A. \& Roberts, P. H. 1995, Nature 377, 203

Käpylä, P. J., Korpi, M. J., \& Brandenburg, A. 2008, A\&A A 491, 353

Kitchatinov, L. L. \& Mazur, M. V. 2000, Solar Phys. 191, 325

Kleeorin, N. \& Rogachevskii, I. 1994, Phys. Rev. E 50, 2716

Kleeorin, N., Moss, D., Rogachevskii, I., Sokoloff, D. 2000, A\&3A 361, L5

Kleeorin, N., Moss, D., Rogachevskii, I., Sokoloff, D. 2002, A\&A 387, 453

Kleeorin, N., Moss, D., Rogachevskii, I., Sokoloff, D. 2003a, A $\& A 4400,9$

Kleeorin, N., Kuzanyan, K., Moss, D., et al. 2003b, A\&A 409, 1097

Köhler, H. 1973, A\&̊ 25, 467

Küker, M., Rüdiger, G., \& Schultz, M. 2001, A\& $A$ 374, 301

Parker, E. N. 1957, Proc. Nat. Acad. Sci. 43, 8

Parker, E. N. 1975, ApJ 198, 205

Parker, E. N. 1984, ApJ 283, 343

Parker, E. N. 1993, ApJ 408, 707 
Seehafer, N. 1996, Phys. Rev. E 53, 1283

Steenbeck, M. \& Krause, F. 1969, AN 291, 49

Roberts, P. H. \& Stix, M. 1972, A\&A A 18, 453

Spiegel, E. A. \& Weiss, N. O. 1980, Nature 287, 616

Subramanian, K. \& Brandenburg, A. 2004, Phys. Rev. Lett. 93, 205001

Subramanian, K. \& Brandenburg, A. 2006, ApJ 648, L71

Thompson, M. J., Christensen-Dalsgaard, J., Miesch, M. S., \& Toomre, J. 2003, ARA\&A 41, 599

Vainshtein, S. I. \& Cattaneo, F. 1992, ApJ 393, 165

Vishniac, E. T. \& Cho, J. 2001, ApJ 550, 752

Yoshimura, H. 1975, ApJS 29, 467

Yousef, T. A. \& Brandenburg, A. 2003, A\&A 407, 7

Yousef, T. A., Brandenburg, A., \& Rüdiger, G. 2003, A\& $A$ 411, 321

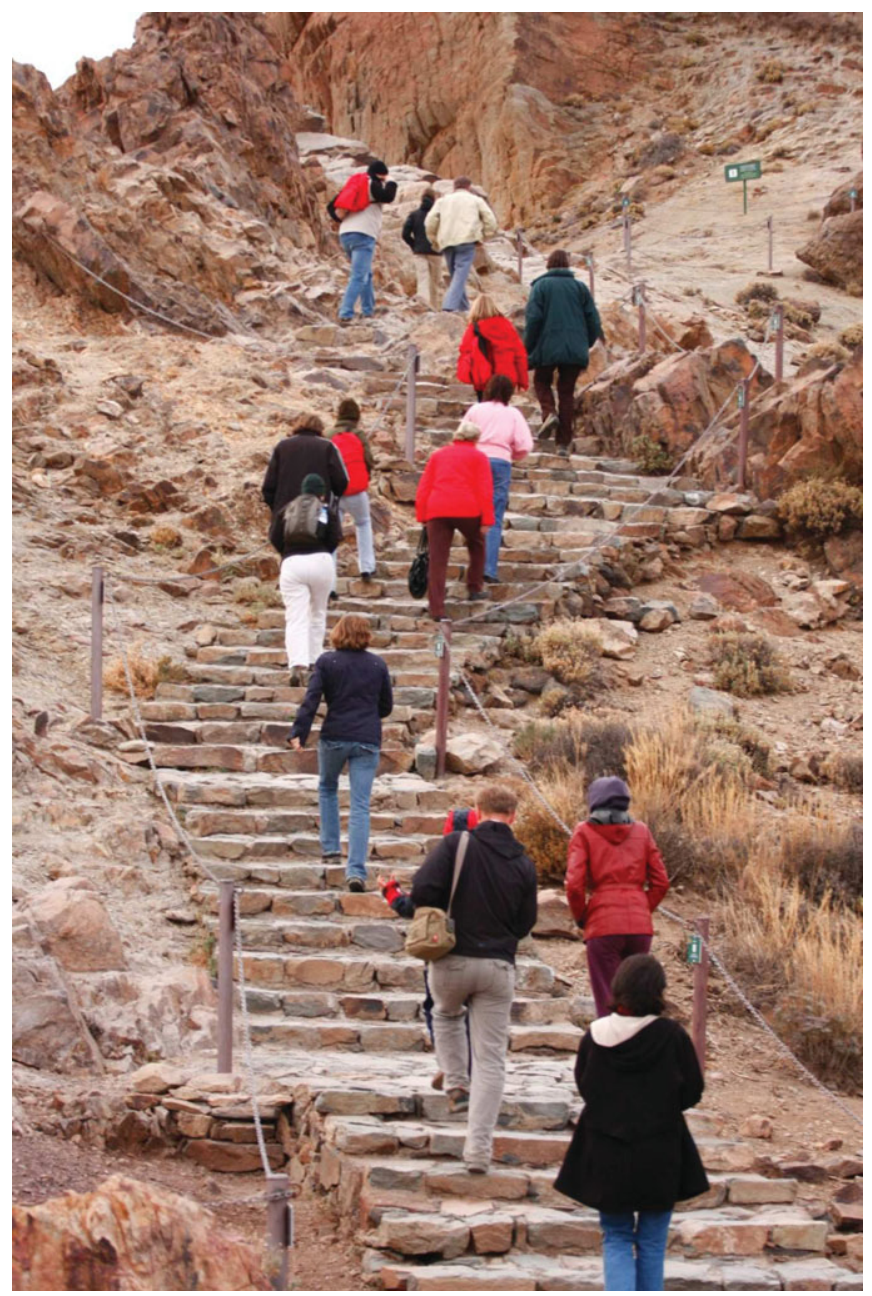

It was a long way up; during the excursion to Teide National Park 
A. Brandenburg

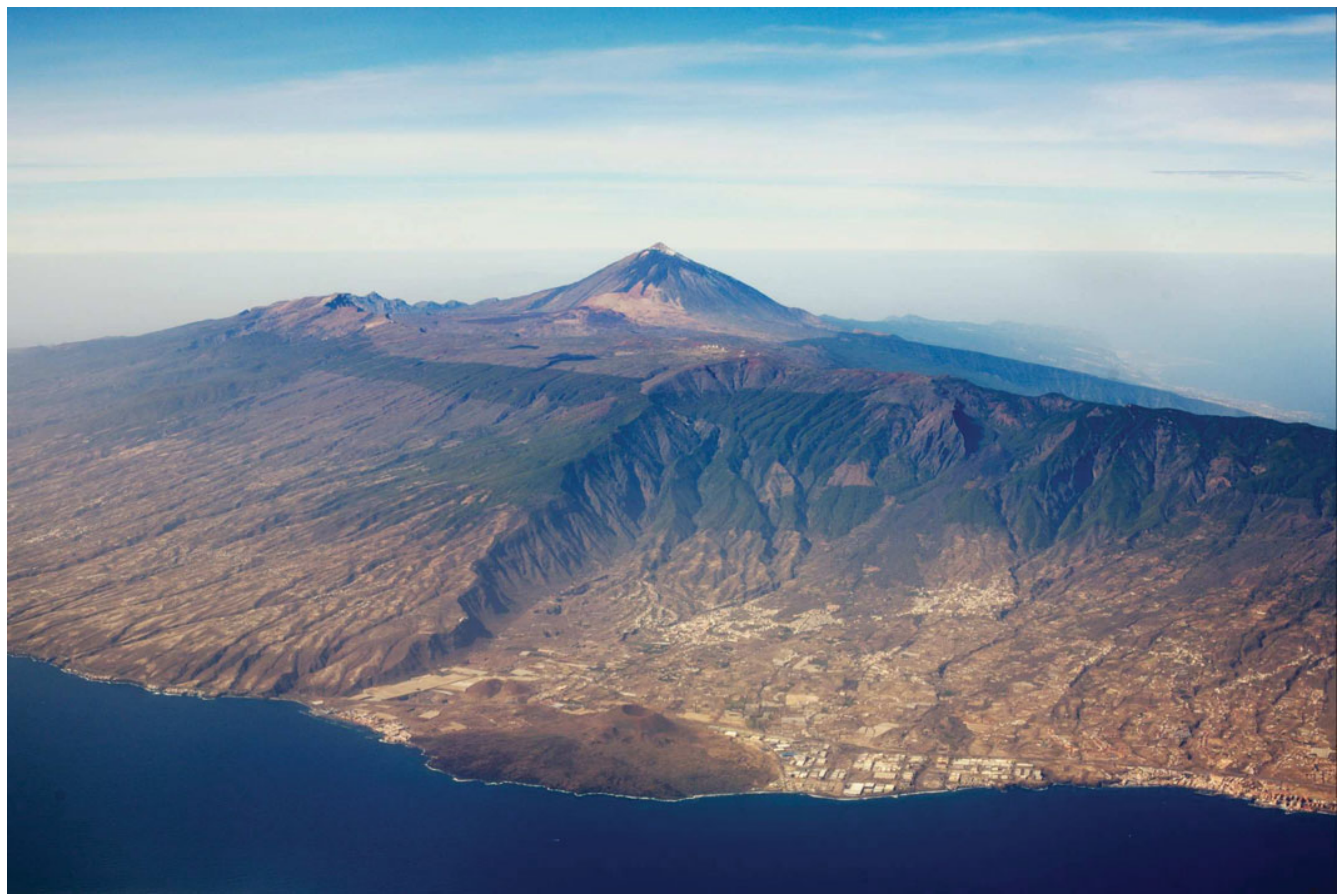

Spectacular Tenerife. Find the Izana observatory on the south-eastern ridge of the Teide!

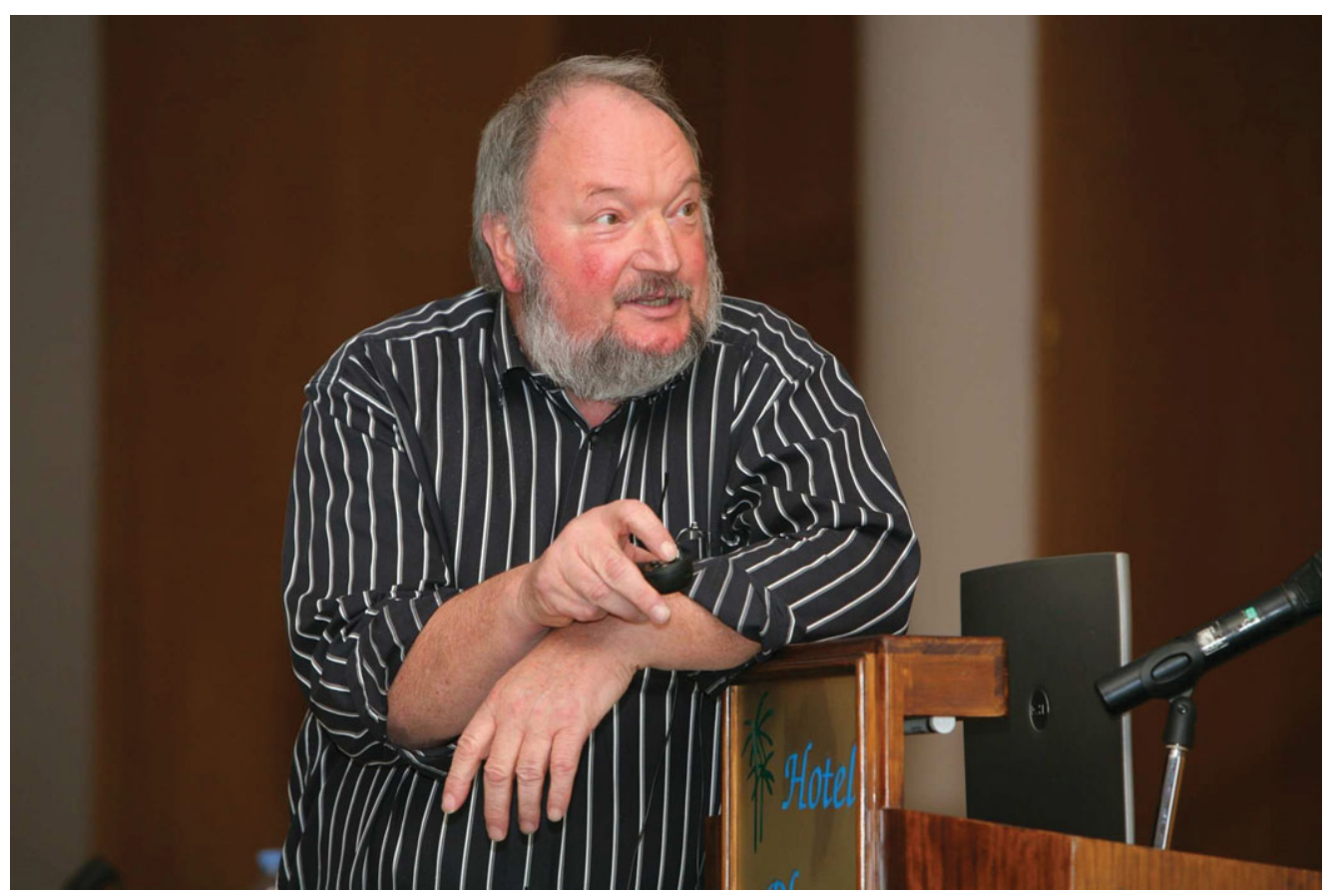

Günther Rüdiger 